\title{
Boron geochemistry in cultured corals and mollusks with independent determinations of internal calcification fluid pool $\mathbf{p H}$
}

ROBERT A EAGLE ${ }^{1}$, MAXENCE GUILLERMIC ${ }^{2}$, LOUISE P. CAMERON ${ }^{3}$, BLANCA ALVAREZ CARAVEO ${ }^{2}$, CLAIRE REYMOND $^{4}$, SAMBUDDHA MISRA ${ }^{5}$, JELLE BIJMA ${ }^{6}$, DIRK DE BEER ${ }^{7}$, HILDEGARD WESTPHAL ${ }^{4}$ AND JUSTIN B. RIES $^{8}$

${ }^{1}$ University of California - Los Angeles

${ }^{2}$ University of California, Los Angeles

${ }^{3}$ Northeastern University

${ }^{4}$ The Leibniz Centre for Tropical Marine Research

${ }^{5}$ Indian Institute of Science

${ }^{6}$ Alfred-Wegener-Institute, Helmholtz-Zentrum für Polar- und Meeresforschung

${ }^{7}$ Max-Planck-Institute for Marine Microbiology

${ }^{8}$ Northeastern University, Boston

Presenting Author: robeagle@g.ucla.edu

The geochemistry of boron in biogenic marine carbonates is widely used as a proxy for seawater $\mathrm{pH}$. However, it is also clear that many marine calcifying organisms actively regulate the $\mathrm{pH}$ of the parent fluid, potentially leading to changes in boron proxy sensitivity to seawater $\mathrm{pH}$ changes. Additionally there are few constraints on whether boron transport to the site of calcification is influenced by biological processes, or if there is boron isotope fractionation between seawater and parent fluids of calcification. Here we present coral skeleton $\mathrm{B} / \mathrm{Ca}$ and $\mathrm{d}^{11} \mathrm{~B}$ data from culturing experiments where the $\mathrm{pH}$ of fluid pockets in the calicoblastic epithelium or corals has been determined using $\mathrm{pH}$ microelectrodes. In addition, we present data from culturing experiments with mollusks where the internal palliol fluid reservoir has been extracted and its $\mathrm{pH}$ and other carbonate system parameters have been determined. In the case of mollusks, we can thereby determine the fractionation between seawater and pallial fluid, and from pallial fluid to shell. For both corals and mollusks, culture temperature and $\mathrm{pCO}_{2}$ was manipulated, allowing the influence of changing environmental parameters on internal fluid pool chemistry and skeleton/shell geochemistry to be assessed. In total, we find that despite positive empirical correlation, an inperfect alignment still exists between known internal fluid pool $\mathrm{pH}$ and carbonate boron geochemistry, when utilizing a canonical application of boron proxy systematics. This may be related to the different timescales recorded by each measurement, or be indicative of the influence of additional complexity in the biomineralisation process. 